\title{
INCLUSION COMPLEXATION OF BENZTHIAZIDE WITH CYCLODEXTRINS IN SOLUTION AND SOLID PHASE
}

\author{
Gamal A. El-Gendy, Ahmed S. Aly and A.M. El-Sayed
}

Department of Pharmaceutics, Faculty of Pharmacy, Assiut University, Assiut, Egypt

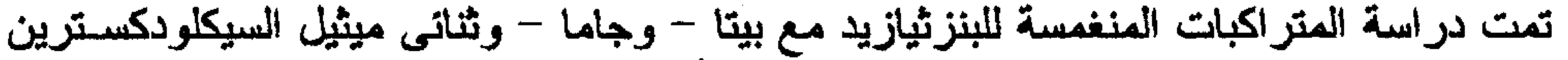

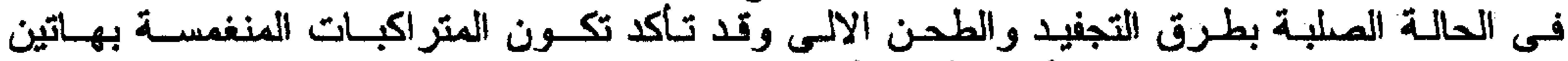

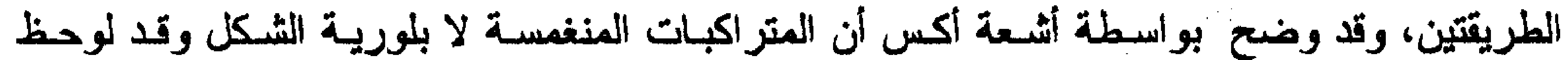

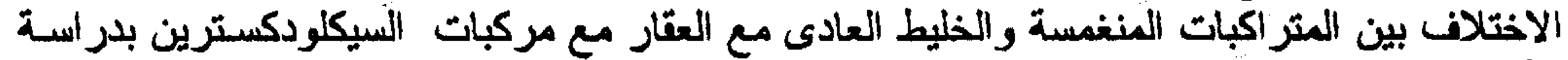

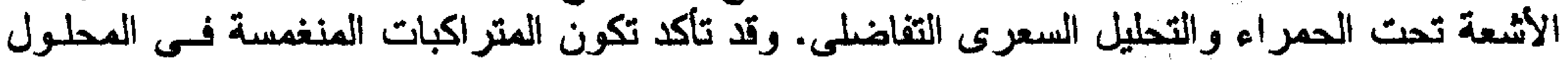

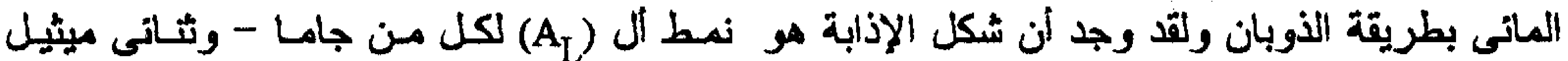

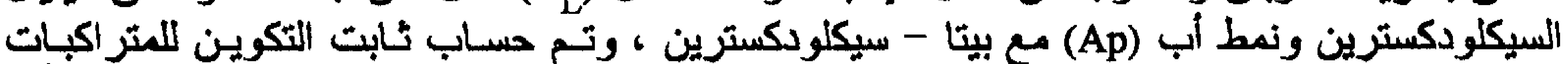

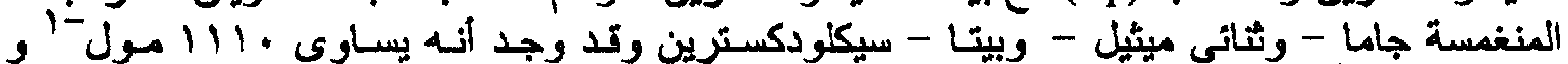

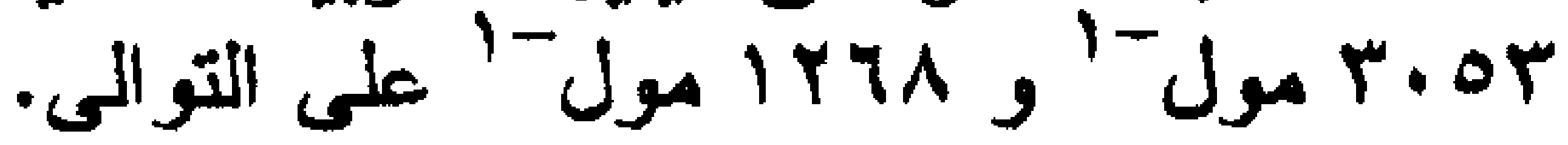

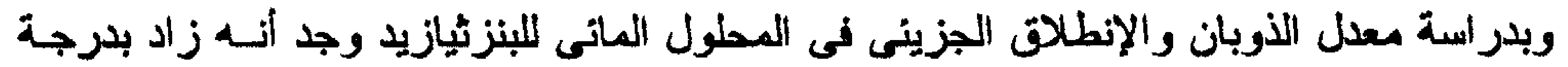

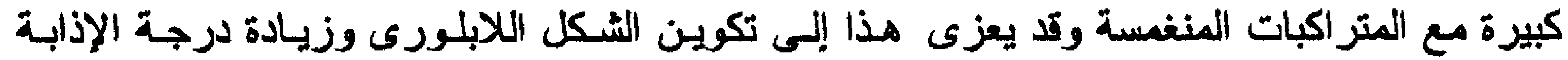

$$
\begin{aligned}
& \text { بطريقنى التجفيد والطحن الالى. }
\end{aligned}
$$

Investigations were made to prepare the inclusion compounds of Benzthiazide (BTZ) with $\beta$-cyclodextrin $(\beta-C D), \gamma$-cyclodextrin $(\gamma-C D)$ and dimethyl- $\beta$-cyclodextrin $(D M C D)$ in solid powdered form by the freeze-drying and the grinding methods.

The freeze drying and grinding methods were successful in obtaining the inclusion compounds of BTZ with the cyclodextrins (CDs). All the inclusion compounds obtained via freeze drying and grinding methods were in powdered form. It was shown by $X$-ray diffractometery that the inclusion compounds obtained by the two methods were amorphous. Clear differences in infrared (IR) absorption spectroscopy and differential scanning calorimetry were observed between the inclusion compounds and the physical mixtures of BTZ-CDs.

Furthermore, inclusion complexation of $B T Z$ with $\gamma-C D, D M C D$ and $\beta-C D$ in aqueous solutions were confirmed by the solubility method. The solubility curves of BTZ- $\gamma-C D$ and BTZ$D M C D$ were typical $A_{L}$ type curves while the solubility curve with $\beta-C D$ can be classified as type $A_{p}$. The apparent stability constants, $K c$, were calculated from the slope and intercept of the solubility diagrams $1110 \mathrm{M}^{-1}, 3053 \mathrm{M}^{-1}$ and $1268 \mathrm{M}^{-1}$ respectively. The dissolution rate of $B T Z$ from the inclusion complexes was much more rapid than that of the BTZ alone. The enhanced dissolution rate may be due to the decrease in crystallinity and the increase in apparent solubility of the drug of the freeze-dried and ground samples, as expected from $\mathrm{X}$-ray diffractometery and solubility study.

\section{INTRODUCTION}

The thiazide drugs are among the most widely used prescription drugs. They are usually the first drug to be employed either in the treatment of mild cases of hypertension or as adjunctive therapy to other drugs. They are more effective agents than loop diuretics in patients who have normal renal function. Benzthiazide is considered the first drug of that group of drugs. The solubility and dissolution are the main problems in starting its effect ${ }^{1,2}$.

Solid inclusion compounds of cyclodextrins (CDs) with many guest molecules have been prepared by coprecipitation ${ }^{3}$, kneeding ${ }^{4}$, freezedrying $^{5}$ and grinding ${ }^{6,7}$ methods. In aqueous

Received in 26/2/1995 \& Accepted in 1/10/1996 
solutions, CDs usually form 1:1 inclusion complexes with guest molecules ${ }^{8,9}$. It is known, however, that inclusion compounds do not necessarily have a stoichiometry of $1: 1^{8}$. In crystals of the heptakis (2,6-di-O-methyl)- $\beta$ cyclodextrin (DMCD) complex with p-nitrophenol, the p-nitrophenol molecules were not included within the cavity but located in the intermolecular space between DMCD molecules ${ }^{10}$, although 1:1 inclusion complexation was observed in aqueous solution ${ }^{11}$.

From the pharmaceutical point of view, if an inclusion compound with $\mathrm{CDs}$ was provided in solid powdered form, it may be more widely used, especially being convenient for oral administration.

The present study was attempted to obtain the inclusion compounds of $B T Z$ with $B-C D$, $\gamma-C D$ and DMCD in solid powdered forms. Freeze-drying method as well as cogrinding method were successful in obtaining the inclusion compounds of the test drug with CDs with high yields.

\section{EXPERIMENTAL}

\section{Materials}

Benzthiazide (BTZ) was purchased from Sigma Chemical Co. (USA) and used without further treatment, $B$-cyclodextrin and heptakis (2,6-di-O-methyl)-B-cyclodextrin (DMCD) were purchased from Toshin Chemical Co. (JapanTokyo), $\gamma$-cyclodextrin was purchased from Nakarai Chemical Co. (Japan-Tokyo). All other chemicals and solvents were of analytical reagent grade. Double distilled water was used through out the study.

\section{Methodology}

\section{1- Preparation of inclusion complex by} grinding

Ground mixtures were prepared using a vibrational mill of Heiko Seisakusho model TJ200 (Rigakudenki Co. Ltd., Tokyo-Japan) which was made of tungsten carbide. Volume of the mill was $140 \mathrm{~cm}^{3}$, and height of the rod was 55 min. Total weight of each sample was about 2.5 gm. The molar ratio of BTZ to CDs was 1:1.

\section{2- Preparation of inclusion compound by lyophilization method}

The inclusion compounds were prepared by the freeze-drying method (T. Nagai method) ${ }^{12}$. Various amounts of a guest compound were dissolved in various aqueous CDs solutions. The sample solutions were kept in liquid nitrogen and then lyophilized according to the following chart 1.

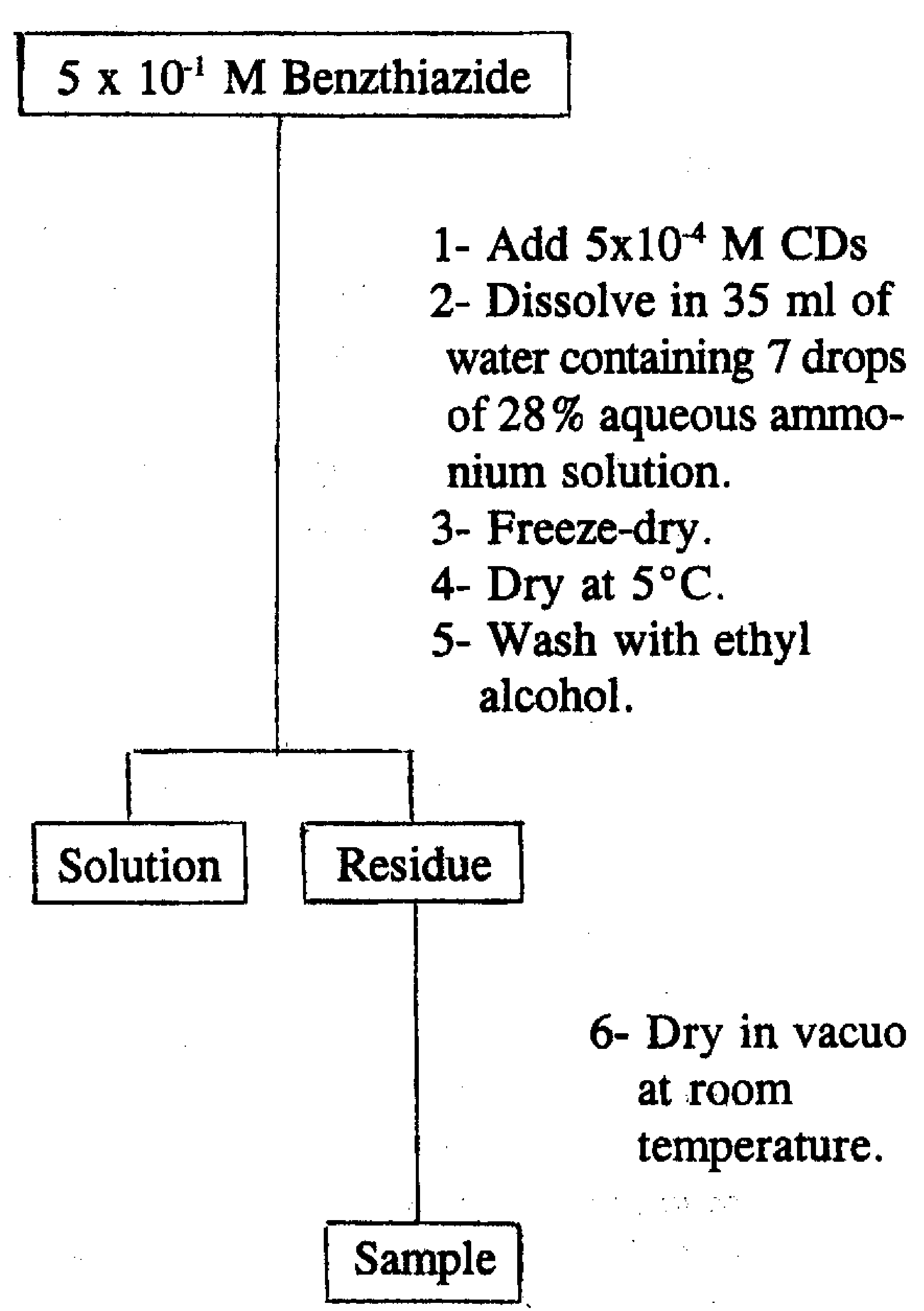

Chart 1: Freeze-drying method for preparation of inclusion compounds.

\section{3- Powder X-ray diffractometery}

A Rigakudenki 2027 diffractometer was used. Measurement conditions were as follows: target, $\mathrm{Cu}$, filter, $\mathrm{Ni}$; voltage, $30 \mathrm{KV}$; current, $5 \mathrm{~mA}$; scintillation counter.

\section{4- Differential scanning calorimetry}

The thermal behaviour of the specimens was measured by a DSC V2.2A Dupont 9900. The measurement were done using the open pan, at a scanning speed of $10^{\circ} \mathrm{C} \mathrm{min}{ }^{-1}$ under 
nitrogen stream, from $25^{\circ} \mathrm{C}$ to $300^{\circ} \mathrm{C}$, sample weight was about $4.0 \mathrm{mg}$.

\section{5- Infrared spectroscopy}

The IR spectra were carried out with Hitachi 295 infrared spectrometer. The $\mathrm{KBr}$ disk method was applied for measurement. The disks were made under a pressure equal to 400 $\mathrm{kg} / \mathrm{cm}^{-2}$.

\section{6- Solubility studies}

Solubility measurements were carried out according to Higuchi and Conners ${ }^{13}$. An excess amount $(100 \mathrm{mg})$ of Benzthiazide was added to aqueous solutions containing CDs at various concentrations, and the solutions were shaken for about $12 \mathrm{hrs}$ at $30^{\circ} \mathrm{C}$. After equilibrium was attained, filtered aliquots were analyzed by spectrophotometry at $296 \mathrm{~nm}$ against a similarly treated blank.

\section{7- Dissolution rate studies}

The dissolution rate studies were done according to the USP XX paddle method in 250 $\mathrm{ml}$ buffer solution $\left(\mathrm{pH} \mathrm{7.2)}\right.$ at $37 \pm 0.5^{\circ} \mathrm{C}$. a certain amount of each sample to BTZ was placed in the dissolution medium. The stirring rate was $50 \mathrm{rpm}$. At appropriate intervals $5 \mathrm{ml}$ of solution was sampled and filtered through a cotton plug, diluted and assayed spectrophotometrically. A correction was applied for the cumulative dilution caused by replacement of the sample by equal volumes of the dissolution medium.

\section{RESULTS AND DISCUSSION}

The X-ray diffraction patterns of BTZ and CDs are shown in Figures 1,2 and 3. Two broad peaks at 4.9 and $7.2 \mathrm{~A}^{\circ}$ are evident in the freeze dried systems. Kurozumi et al. ${ }^{12}$ reported the presence of similar diffraction peaks at interplanar distances 7.2-7.7 and 4.8-50 $\mathrm{A}^{\circ}$ in freeze-dried inclusion complexes of 13 different drugs. Earlier Takeo and Kuge ${ }^{14}$ observed two such peaks in B-CD-propyl alcohol and B-CDanthracene complexes. Both groups of workers attributed these broad peaks to the cylindrical structure of B-CD, the "guest" molecule, being included in the "host". These results suggest that the freeze-dried BTZ-CDs systems may be inclusion complexes. Alternatively, the data might be explained sololy in terms of formation of amorphous drug on freeze drying with CDs. The BTZ-CDs freeze dried systems as well as the mechanically grinding systems exhibited lower crystallinity than the corresponding mechanically mixed systems (physical mixtures). In the mean time characteristic BTZ peaks were existed. The X-ray scans of BTZ with DMCD and $\gamma-C D$ were similar to corresponding drug- $B$ CD systems.

BTZ crystals melted giving an endothermic peak at $237^{\circ} \mathrm{C}$ which was also present in the physical mixture at $226^{\circ} \mathrm{C}$. The disappearance of the endothermic peak, due to the melting of the freeze-dried BTZ-B-CD system, suggests the presence of a solid state complex (Fig. 4). Figure 5 shows DSC curves of BTZ with DMCD in physical mixtures, freeze-drying and grinding system (molar ratio 1:1). One endothermic peak was observed in the physical mixture around $236^{\circ} \mathrm{C}$ due to the fusion of BTZ crystals. In the freeze-drying system, a new exothermic peak around $138^{\circ} \mathrm{C}$, and another peak around $144^{\circ} \mathrm{C}$ with ground mixture, were interpreted to be due to the crystallization of BTZ-DMCD complex. There are also endothermic peak at $222^{\circ} \mathrm{C}$ for ground mixture and at $223^{\circ} \mathrm{C}$ for freeze-drying system, which represent the melting of inclusion complexes. These results indicate the possibility of inclusion complex formation by the grinding and freezedrying techniques. The DSC patterns of BTZ- $\gamma-$ $\mathrm{CD}$ were similar to corresponding BTZ-B-CD systems.

Molecular states of BTZ molecules in BTZCDs samples were studied by means of IR spectroscopy. Figure 6 shows the IR spectrum of the drug in the range of $1400-1700 \mathrm{~cm}^{-1}$. The spectrum of BTZ crystals was characterized by $\mathrm{C}=\mathrm{C}$ and $\mathrm{NH}_{2}$ stretching bands at 1499 and $1589 \mathrm{~cm}^{-1}$, respectively. These stretching bands of the ground and freeze dried samples were shifted to different frequency compared with the BTZ crystals and appeared at 1506 and 1579 $\mathrm{cm}^{-1}$ with 1:1 BTZ-DMCD freeze-dried sample and at $1503 \mathrm{~cm}^{-1}$ while the other peak disappeared with 1:1 BTZ-DMCD ground mixture. The results indicated that BTZ 

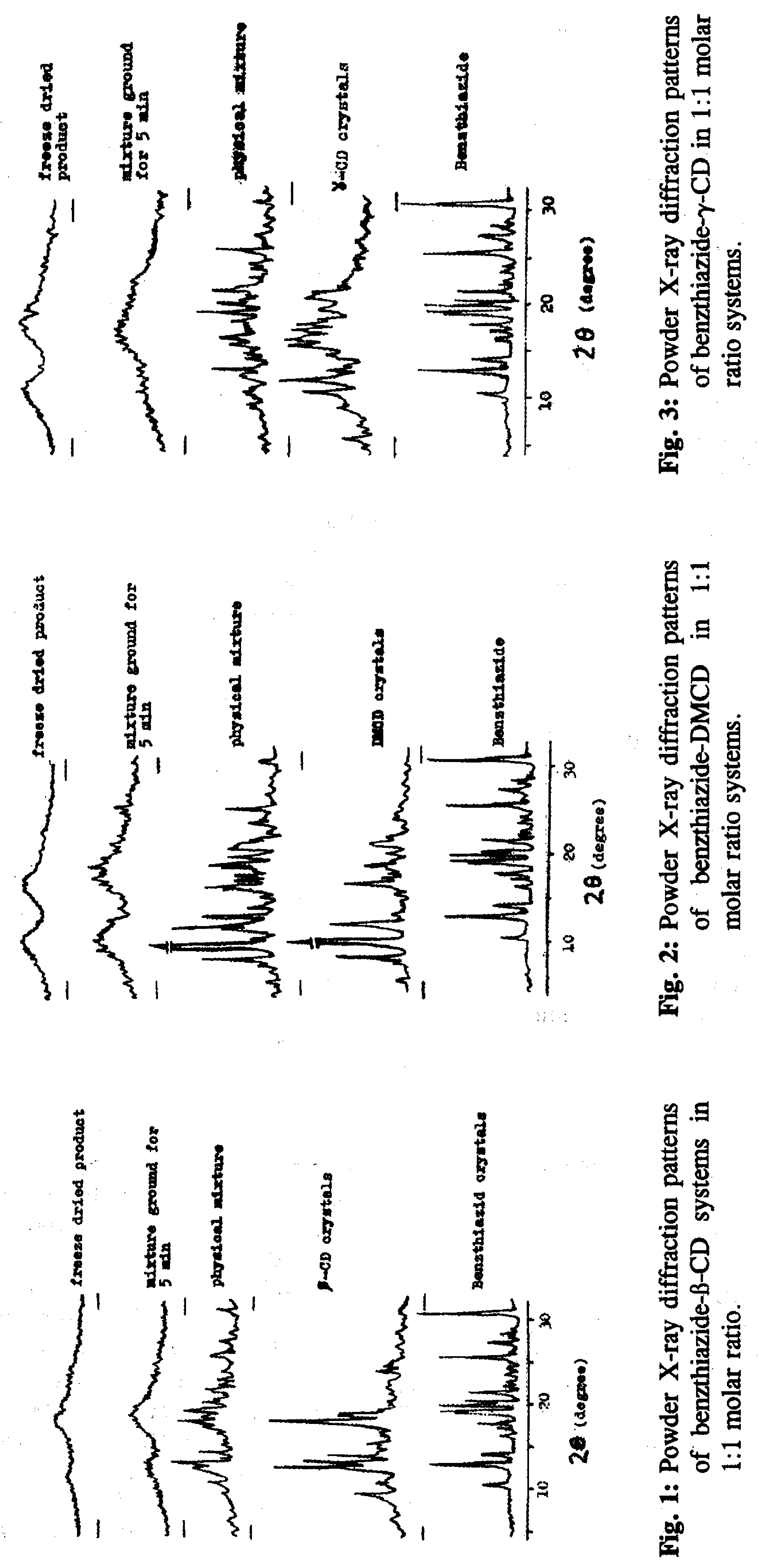

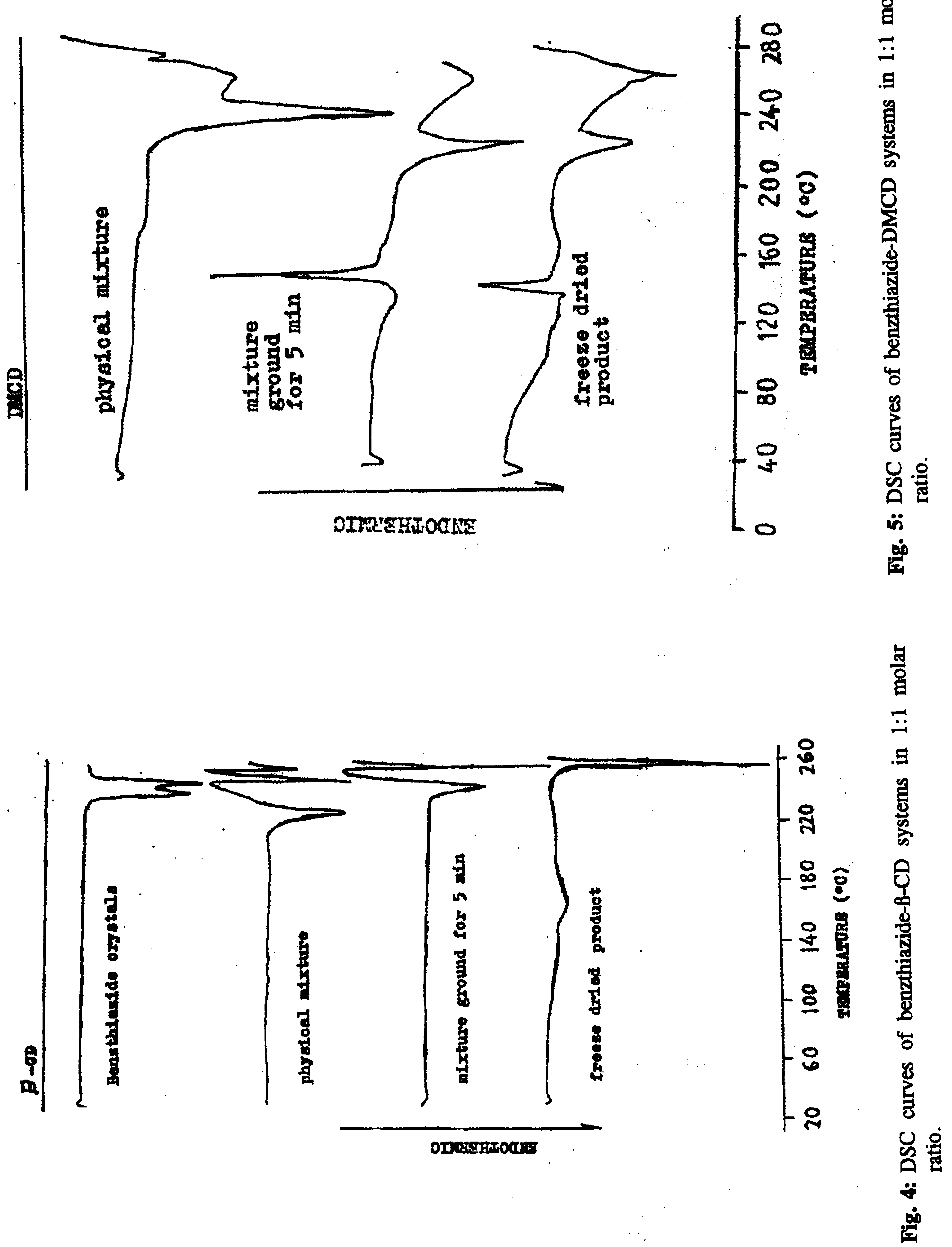


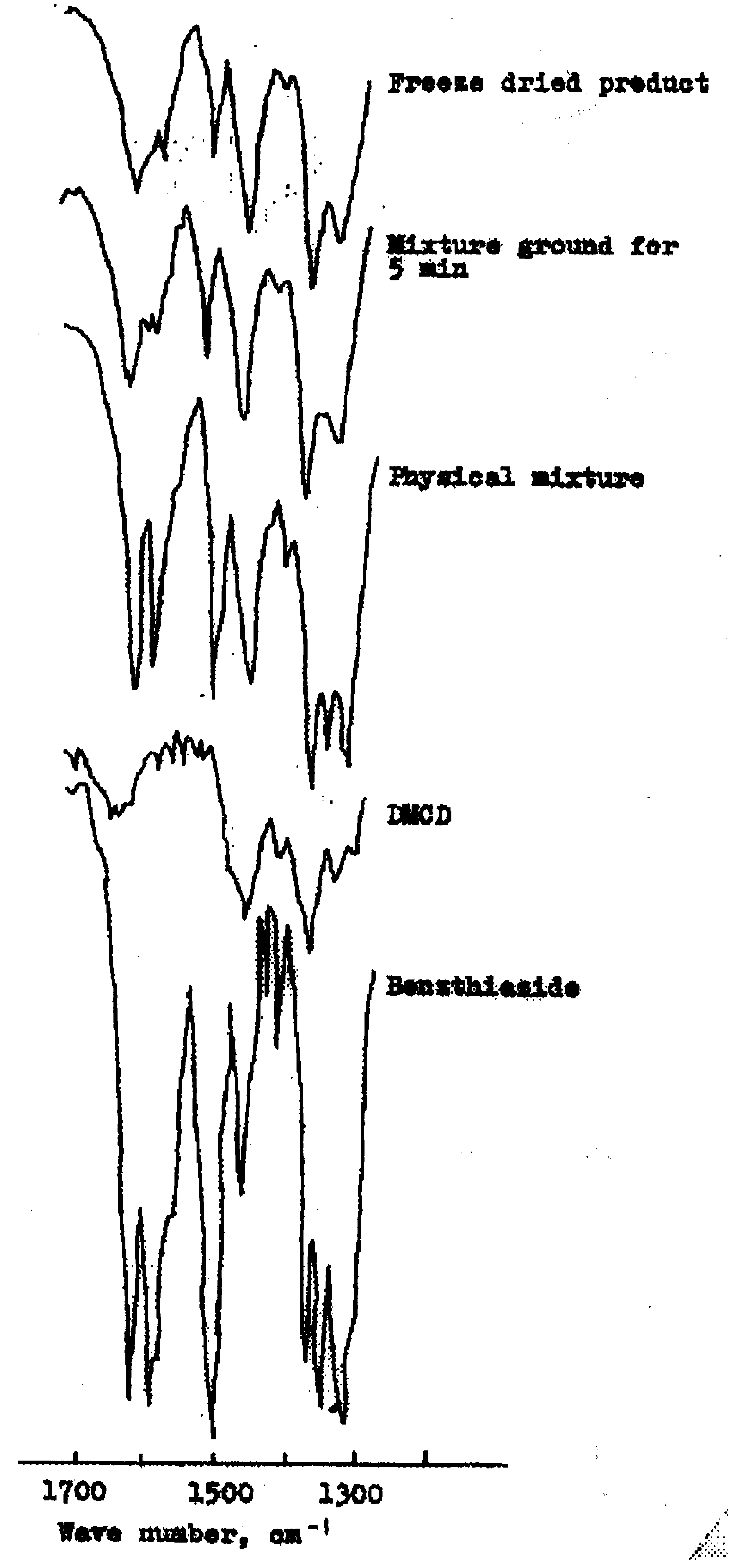

Fig. 6: IR spectra of benzthiazide-DMCD in 1:1 molar ratio systems.

molecules in the freeze-dried and ground samples existed in a state closely similar to the included state within the DMCD cavity. Similar results were obtained with other CDs.
Inclusion complexation in solution (Fig. 7) shows the phase solubility diagrams in water of BTZ with various concentration of CDs. The solubility $\left(1 \times 10^{-5} \mathrm{M}\right)$ of BTZ increased linearly with increase in CDs concentration, showing $A_{L}$ type solubility diagrams with $\gamma-C D$ and DMCD, and Ap type with $B-C D$ according to Higuchi and Connors ${ }^{13}$. Assuming that a 1:1 complex is initially formed, the apparent stability constant $(\mathrm{Kc})$ of the complexes was calculated in terms of the following equation ${ }^{12}$,

$$
\mathrm{Kc}=\{\text { slop }\} / \text { intercept (1-slop) }\}
$$

using the slope and intercept of the straight lines of the diagram.

The Kc values of the complexes were in the order of $\gamma$-CD $\left(1110 \mathrm{M}^{-1}\right)<\beta-C D\left(1268 \mathrm{M}^{-1}\right)$ $<$ DMCD $\left(3053 \mathrm{M}^{-1}\right)$. The inclusion ability of B-CD toward BTZ was enhanced by the introduction of the relatively hydrophobic methyl group into the host molecule as in case of $\mathrm{DMCD}^{15}$.

The dissolution rate profiles of complexes, physical mixture and intact BTZ were studied (Fig. 8). The freeze-dried complex exhibits a faster drug dissolution rate than intact BTZ, where the dissolution rate of intact drug was extremely slow. The enhancing dissolution rate of the complexes may be due to the enhanced solubility in water (Fig. 7) and amorphous character (Figs. 1,2 and 3) of the complexes.

\section{Conclusion}

From these results, the freeze-drying method and grinding method were suitable for obtaining inclusion complex of BTZ with CDs in powdered form. The inclusion complexation of BTZ with CDs were confirmed by X-ray diffractometry, DSC scans and IR.

Thus, the present study indicates that the aqueous solubility and dissolution rate of BTZ can be significantly increased by forming an inclusion complex with CDs. This enhancement in solubility brought about by complexation, may be of potential use in developing a suitable oral dosage form of BTZ. 

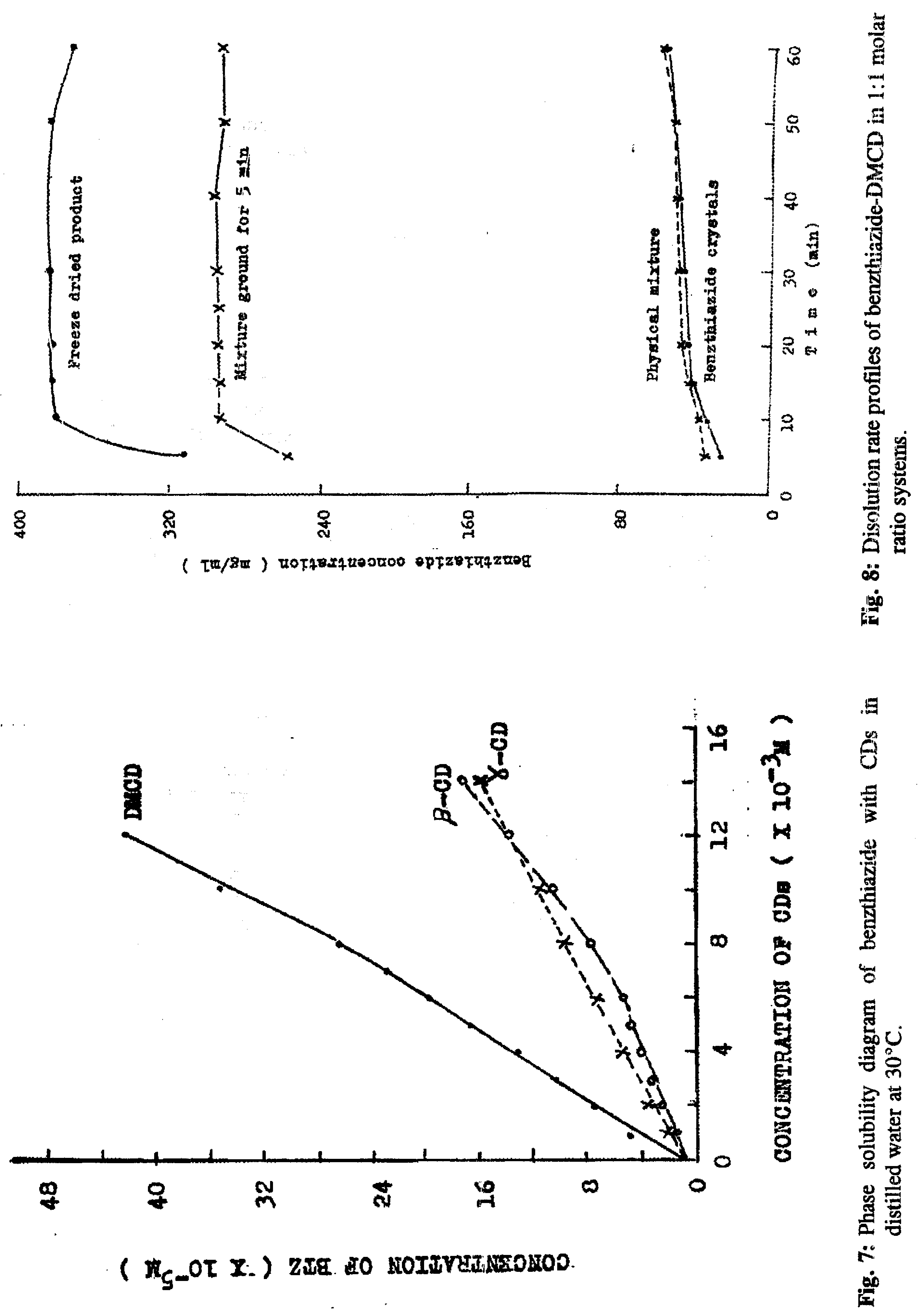


\section{REFERENCES}

1- A.R.Gennaro, Editor; "Remingtons Pharmaceutical Sciences", 18th End, Mack Publishing Co., Easton, Pennsylvania, p. 934 (1990).

2- A.G.Gilman, T.W.Rall, A.S.Nies and P.Taylor, Editors; "The Pharmacological Basis of Therapeutics", 8th Edn, Pergmon Press Inc., Singapore, p. 785 (1991).

3- F.M.Andersen and H.Bandgoard, Inclusion complexation of metronidazole benzoate with B-cyclodextrin and its depression of anhydate-hydrate transition in aqueous suspensions, Int. J. Pharm., 19, 189 (1984).

4- M.Kikuchi, F.Hirayama and K.Uekama, Improvement of oral and rectal bioavailabilities of carmfour by methylated B-cyclodextrin complexations, Int. J. Pharm., 38, 191 (1987).

5- T.Oguchi, M.Okada, E.Yonemochi, K.Yamamoto and Y.Naka, Freeze-drying of drug-additive binary systems, III. Crystallization of $\alpha$-cyclodextrin inclusion complex in freezing process, Int. J. Pharm., 61, 27 (1990).

6- Y.Nakai, K.Yamamoto, K.Terada and K.Akimoto, The dispersed states of medicinal molecules in ground mixtures with $\alpha$ - and $\beta$-cyclodextrins, Chem. Pharm. Bull., 32, 685 (1984).

7- G.A.El-Gendy, M.A.El-Gendy, Heptakis (2,6-di-O-methyl)-B-cyclodextrin complexation with Glutethimide, Eur. J. Pharm. Biopharm., 39, 249 (1993).

8- M.L.Bender, M.Komiyama, Cyclodextrin chemistry, Springer-Verlag, Berlin, New York (1978).
9- J.Szejtli, Cyclodexrin and their inclusion complexes, Akademiai Kiado, budapest (1982).

10- K.Harata, The structure of the cyclodextrin complex. XXI. Crystal structures of heptakis (2,6-di-O-methyl)-B-cyclodextrin complexes with $p$-iodophenol and $p$ nitrophenol, Bull. Chem. Soc. Jap., 61, 1939 (1988).

11- Y.Nakai, K.Yamamoto, K.Terada, T.Oguchi and G.Nakamura, Molecular interactions between heptakis (2,6-di-Omethyl)-B-cyclodextrin and p-nitrophenol in solutions, Yakugaku Zasshi, 107, 802 (1987).

12- M.Kurozumi, N.Nambu and T.Nagai, Inclusion compounds of non-steroidal antiinflammatory and other slightly water soluble drugs with $\alpha$ - and B-cyclodextrins in powdered form, Chem. Pharm. Bull., 23, 3062 (1975).

13- T.Higuchi and K.A.Connors, Phasesolubility techniques, Adv. Anal. Chem. Instr., 4, 117 (1965).

14- K.Takeo, T.Kuge, Complexes of starchy materials with organic compounds III: Xray studies on amylose and cyclodextrin complexes, Agr. Biol. Chem., 33, 1174 (1969).

15- A.Yoshida, M.Yamamoto, T.Itoh, T.Irie, F.Hirayama and K.Uekama, Utility of 2hydroxypropyl-B-cyclodextrin in an intramuscular injectable preparation of Nimodipine, Chem. Pharm. Bull., 38, 176 (1990). 